\title{
Regulation of autophagy by protein post-translational modification
}

\author{
Willayat Yousuf Wani ${ }^{1,2}$, Michaël Boyer-Guittaut ${ }^{3}$, Matthew Dodson ${ }^{1,2}$, John Chatham ${ }^{1,2}$, Victor Darley-Usmar ${ }^{1,2}$ \\ and Jianhua Zhang ${ }^{1,2,4}$
}

Autophagy is a lysosome-mediated intracellular protein degradation process that involves about 38 autophagy-related genes as well as key signaling pathways that sense cellular metabolic and redox status, and has an important role in quality control of macromolecules and organelles. As with other major cellular pathways, autophagy proteins are subjected to regulatory post-translational modification. Phosphorylation is so far the most intensively studied post-translational modification in the autophagy process, followed by ubiquitination and acetylation. An interesting and new area is also now emerging, which appears to complement these more traditional mechanisms, and includes O-GlcNAcylation and redox regulation at thiol residues. Identification of the full spectrum of post-translational modifications of autophagy proteins, and determination of their impact on autophagy will be crucial for a better understanding of autophagy regulation, its deficits in diseases, and how to exploit this process for disease therapies.

Laboratory Investigation (2015) 95, 14-25; doi:10.1038/labinvest.2014.131; published online 3 November 2014

Post-translational modifications of proteins have a key role in determining protein structure, destination, activity, and function. These modifications include, but are not limited to, phosphorylation to regulate catalytic activity and proteinprotein interactions, glycosylation to ensure proper protein folding, ubiquitination to signal degradation, and lipidation to enable insertion into phospholipid membranes. ${ }^{1-4}$ The ability of the cell to maintain these modifications is governed by a variety of factors, such as nutrient availability and proper organellar function, with changes to normal homeostasis resulting in changes to protein function. While many post-translational modifications are tightly regulated, increased cellular stress can cause the system to break down, or introduce non-specific post-translational modifications that do not occur during physiological conditions. Autophagy is a highly regulated multi-step process directing the formation of autophagosomes and the degradation of their content by the lysosomes. ${ }^{5,6}$ Autophagy is regulated by a number of signaling cascades involving multiple cellular kinases (AMPK, AKT) that regulate MTORC1 phosphorylation, the central key protein in autophagy regulation. The execution of autophagy requires the function of several protein complexes and pathways, including the MTORC1 complex, the ULK1/ATG1 complex, the LC3 conjugation pathway, as well as the PI3K class III/VPS34 (phosphatidylinositol 3-kinase/vacuolar protein sorting 34) complex (Figure 1). The activities of these complexes and pathways are highly dependent on regulatory post-translational modifications of autophagy proteins, which is the subject of this review.

\section{REGULATION OF AUTOPHAGY BY PHOSPHORYLATION MTORC1 Phosphorylation Inhibits Autophagy}

Phosphorylation is a post-translational modification that inserts a phosphate group onto serine, threonine, and tyrosine residues, changing protein conformation, activity, and protein-protein interactions. ${ }^{4}$ So far, regulation of autophagy by phosphorylation of autophagy proteins is the best understood and the most intensively studied posttranslational modification.

One of the best-characterized autophagy regulators is MTOR (mammalian target of rapamycin). MTORC1 is part of a complex comprising RAPTOR (regulatory-associated protein of MTORC1), MLST8/G $\beta$ L (mammalian lethal with Sec13 protein $8 / G$ protein subunit $\beta$ like), DEPTOR (DEP-domain-containing and MTOR-interactive protein),

${ }^{1}$ Center for Free Radical Biology, University of Alabama at Birmingham, Birmingham, AL, USA; ${ }^{2}$ Department of Pathology, University of Alabama at Birmingham, Birmingham, AL, USA; ${ }^{3}$ Laboratoire de Biochimie, EA3922, SFR IBCT FED4234, Sciences et Techniques, Université de Franche-Comté, Besançon Cedex, France and ${ }^{4}$ Department of Veterans Affairs, Birmingham VA Medical Center, Birmingham, AL, USA

Correspondence: Dr J Zhang, PhD, Department of Pathology, University of Alabama at Birmingham, Biomedical Research Building II, 901 19th Street South, Birmingham, AL 35294, USA.

E-mail: zhanja@uab.edu

Received 18 June 2014; accepted 14 August 2014 


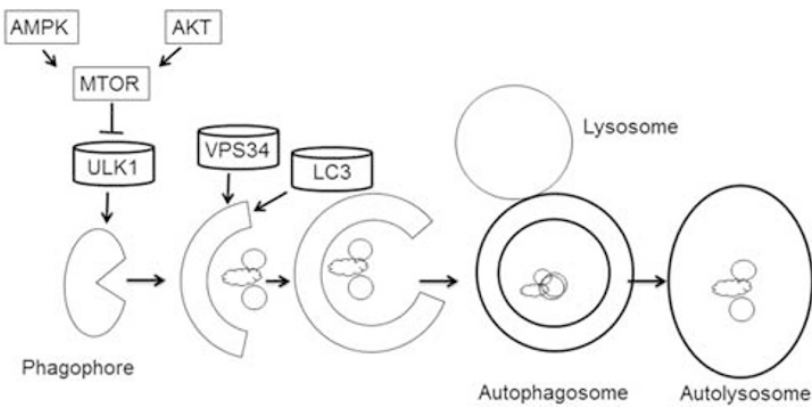

Figure 1 Overview of autophagy. Autophagy is a lysosome-mediated intracellular degradation activity by which double membrane vesicles engulf proteins and organelles in a process that is generally regulated by MTOR and VPS34 complexes and involves insertion of LC3 into autophagosomal membranes.

and PRAS40 (proline-rich AKT substrate of $40 \mathrm{kDa}$ ) (Figure 2). ${ }^{7}$ Under normal conditions, MTORC1 is phosphorylated and active, and negatively phosphorylates the serine/threonine kinase ULK1/ATG1 (Unc51-like kinase 1) complex as well as ATG13, two members of the ULK1/ATG1 complex that also includes FIP200/ATG17 (focal adhesion kinase (FAK) family interacting protein of $200 \mathrm{kDa}$ ) and ATG101. Following a cellular stress (eg, hypoxia or nutrient depletion), MTORC1 is inactivated, leading to the release of the ULK1/ATG1 complex, its dephosphorylation, and the subsequent activation of ULK1 kinase activity. ULK1 then phosphorylates itself and its partners, ATG13 and FIP200, leading to the activation of autophagy (Figure 2). ${ }^{7,8}$

\section{Regulation of MTORC1 by AMPK and AKT}

Being the key regulator of autophagy initiation, MTORC1 activity is regulated by multiple upstream signaling pathways. One of the main signaling pathways is the AKT/AMPK (AMP-activated protein kinase) cascade. AMPK is the cellular energy sensor since it can be activated following an increase in the AMP/ATP ratio due to a nutrient or hypoxic stress. AMPK is known to be phosphorylated by the serine/threonine kinase LKB1 (liver kinase B1) and to phosphorylate Tuberous Sclerosis Complex 2 (TSC2), a GTPase activating protein (GAP) domain-containing protein, which inactivates the Rheb GTPase, a direct activator of MTORC1 (Figure 2). More recently, AMPK has also been shown to directly phosphorylate RAPTOR, another MTORC1 complex protein, to fully inactivate MTORC1 during an energetic stress. ${ }^{9}$ In addition to regulation of MTORC1 activities, AMPK can directly phosphorylate ULK1 to regulate mitophagy and mitochondrial homeostasis, thereby creating a parallel pathway for ULK1 activation. ${ }^{10}$ Under glucose starvation, the activated AMPK inhibits MTORC1 to relieve Ser757 phosphorylation, leading to ULK1-AMPK interaction. AMPK then phosphorylates ULK1 on Ser317 and Ser777, activates ULK1 kinase, and eventually leads to autophagy induction. ${ }^{11}$
On the other hand, the PI3K class I (PI3KI) can negatively regulate the autophagy process via the synthesis of phosphatidylinositol-3,4-diphosphate (PI-3,4-P2) and phosphatidyl-inositol-3,4,5-trisphosphate (PI-3,4,5-P3) and the recruitment of Pleckstrin Homology $(\mathrm{PH})$ domain-containing proteins such as 3-phosphoinositide-dependent protein kinase-1 (PDK1) or its downstream target, AKT, which in turn activates MTORC1, leading to the inhibition of autophagy induction. ${ }^{12}$ AKT can also directly phosphorylate PRAS40 to activate MTORC1 (Figure 2). ${ }^{13,14}$

\section{The Regulation of BECN1/VPS34 Complex by Phosphorylation}

In mammalian cells, the PI3K class III/VPS34 interacts with P150 (PI3-kinase P150 subunit)/VPS15 and BECN1 (coiled-coil myosin-like BCL2-interacting protein 1)/ATG6. ${ }^{15}$ This kinase induces the synthesis of phosphatidylinositol 3 phosphate (PI3P), which participates in the formation of the phagophore and the recruitment of other partner proteins such as DFCP1 (double FYVE domain-containing protein), ${ }^{16}$ the WIPI family proteins (WD-repeated protein interacting with phosphoinositides), ${ }^{16}$ and ALFY, which contains a FYVE domain. ${ }^{17}$ Several proteins have been described to interact with the PI3KIII complex and regulate, positively or negatively, the activity of this complex. These include: Beclin1-associated autophagy-related key regulator (BARKOR)/ ATG14; ultraviolet irradiation resistance-associated gene (UVRAG)/VPS38; BAX-interacting factor 1 (BIF1); activating molecule in Beclin1-regulated autophagy 1 (AMBRA1) and RUBICON. The $\beta$ cell lymphoma 2 (BCL2) and BECN1 interaction is regulated by phosphorylation. ${ }^{18,19}$ Under nutrient excess conditions, BCL2 binds BECN1 and inhibits autophagy, but after nutrient depletion, BCL2 is phosphorylated by the kinase c-Jun N-terminal protein kinase 1 (JNK1) on three distinct sites, inducing its release from the PI3KIII complex and autophagy activation (Figure 2). ${ }^{19}$ BECN1 can also be phosphorylated by the death-associated protein kinase (DAPK) causing dissociation of the BCL2-BECN1 complex, ${ }^{20}$ and its phosphorylation by $\mathrm{AKT}$ and protein kinase $\mathrm{B}$ (PKB). These post-translational modifications then lead to the inhibition of autophagy by favoring the interaction of BECN1 with 14-3-3 and the vimentin intermediate filament protein $1 .^{21}$

\section{The Regulation of ATG9 by Phosphorylation}

ATG9 is, unlike most of the ATG proteins, a trans-membrane protein localized at the phagophore assembly site (PAS), as well as detected at peripheral sites. ${ }^{22}$ This protein, which interacts with WIPI-1/ATG18, has been described to travel from the trans-Golgi network and late endosomes toward the PAS and allow the transport of phospholipids or membranes. ${ }^{23}$ More interestingly, this relocalization has been shown to be dependent on the ULK1/ATG1 complex and the PI3KIII/VPS34 kinase activity (Figure 2). ${ }^{24}$ The mechanism by which ATG9 travels through the cell during the autophagy process is still unclear, but a recent study in yeast 


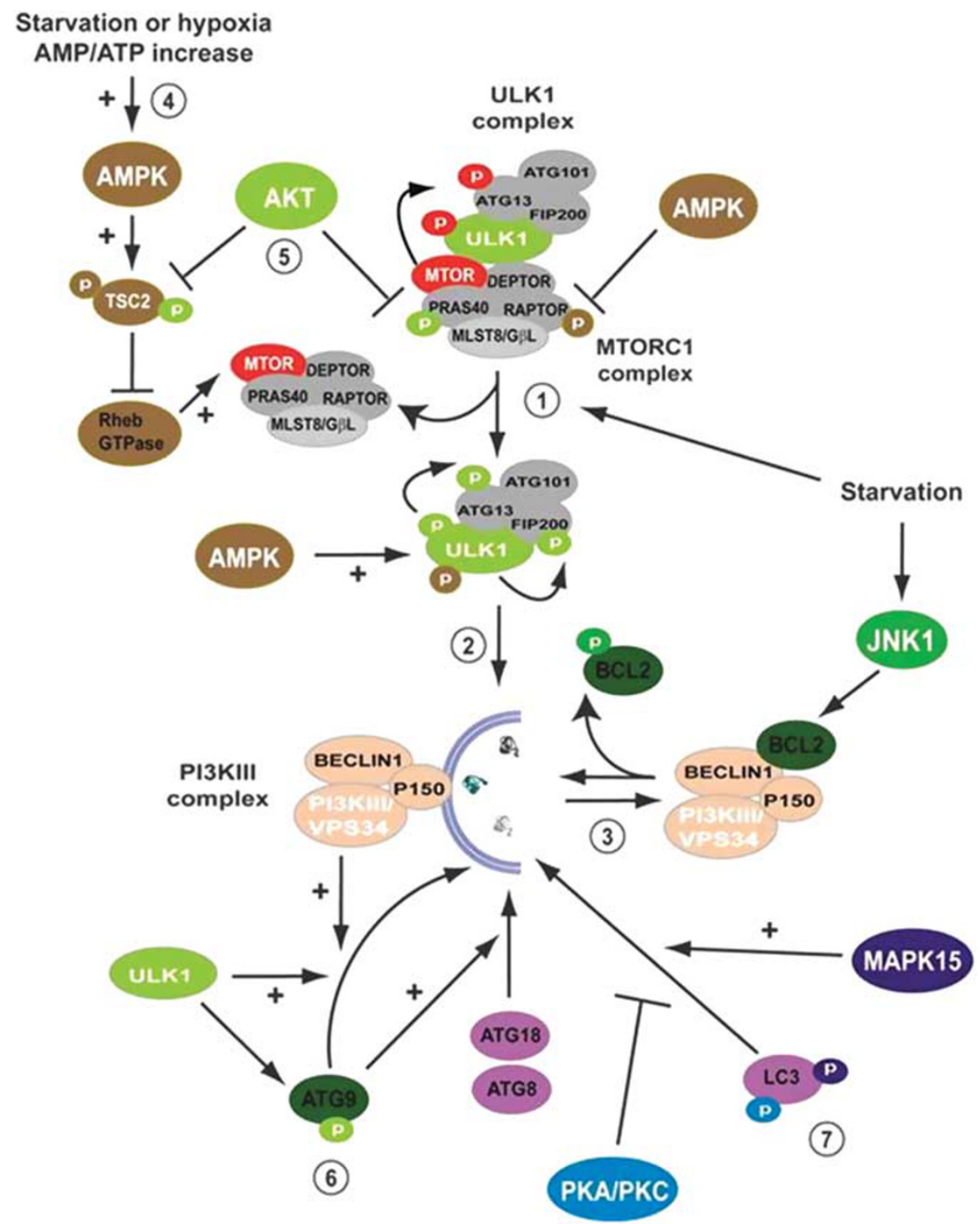

Figure 2 Phosphorylation of autophagy proteins. Under normal conditions, the phosphorylated active MTORC1 complex interacts with and inhibits the ULK1/ATG1 complex. Following autophagy induction in response to starvation, or rapamycin exposure, the MTORC1 complex releases the ULK1/ATG1 complex leading to its activation and its auto-phosphorylation on ULK1, FIP200, and ATG13 (1). The ULK1 complex then recruits the PI3KIII complex to induce the formation of the phagophore (2). This step is also regulated by the BECN1/ATG6-BCL2 interaction and the JNK1 kinase (3). Upstream AMPK activates (4), and AKT inhibits (5), the autophagy process. ATG9 (6) and LC3 (7) are also regulated by phosphorylation.

demonstrated that ULK1/ATG1 directly phosphorylates ATG9, and that this phosphorylation event is necessary for the later recruitment of ATG8 and WIPI-1/ATG18 to the PAS to further activate autophagosome formation. ${ }^{25}$

\section{The Regulation of ATG8/LC3 Family Proteins by Phosphorylation}

Several phosphorylation modifications have also been described to regulate downstream effectors of the autophagy pathway. The interaction of GABARAP and GABARAPL2/ GATE-16 with ULK1/ATG1 in neurons has been suggested to have an important role in vesicle transport and neuronal elongation. ${ }^{26}$ Subsequently, it was found that LC3 (microtubuleassociated protein-light chain 3 ) is phosphorylated by protein kinase A (PKA) and protein kinase C (PKC). ${ }^{27,28}$ PKA phosphorylation of LC3 inhibits its lipidation and incorporation into autophagosomes, resulting in inhibition of autophagy, whereas PKC inhibits the lipidation of LC3, but a phosphorylation mutant of the target site does not significantly change autophagic flux, suggesting that the PKC regulation of autophagy does not involve LC3 (Figure 2). ${ }^{27,28}$ In addition to LC3, GABARAP and GABARAPL1 can also be phosphorylated by the mitogen-activated protein kinase 15 (MAPK15)/ ERK8, which induces their lipidation, resulting in subsequent autophagosome formation and SQSTM1 degradation. ${ }^{29}$ MAPK15 is induced in response to starvation, where it localizes into autophagosomes and prevents the inhibitory phosphorylation of LC3 by PKA. ${ }^{29} \mathrm{~A}$ recent study also demonstrated the 
interaction between ULK1, ATG13, and FIP200 with ATG8 proteins via their LC3-interacting region (LIR) domain, where the ATG8 proteins were suggested to act as a scaffold for the ULK1 complex recruitment onto the autophagosomes. ${ }^{30}$

\section{The Regulation of SQSTM1/p62, ATG29, and ATG31 by Phosphorylation}

SQSTM1 (sequestosome 1)/p62 has also been described to be phosphorylated in its ubiquitin-associated (UBA) domain at Ser403 by the casein kinase 2 (CK2). ${ }^{31}$ This modification increases p62 affinity for its ubiquitinated substrates to activate their degradation. In yeast, a recent study established that ATG29, which is part of the trimeric complex ATG17ATG31-ATG29, is phosphorylated and that this modification is essential for its association with the scaffold protein ATG11, and the recruitment of the ATG17-ATG31-ATG29 complex to the PAS and the recruitment of downstream ATG proteins including ATG1. ${ }^{32}$ Interestingly, the phosphorylation of ATG29 induces a conformational change leading to a change to the localization of a C-terminal inhibitory peptide that allows the activation of the $\mathrm{N}$-terminal functional domain, although the kinase that is responsible for the phosphorylation of ATG29 has not been found. It is noteworthy that ATG31, another member of this complex, is also a phosphoprotein, but the significance of its modification is still unknown. ${ }^{33}$

\section{Phosphorylation of Proteins Involved in Mitophagy}

Autophagy of the mitochondria, or mitophagy, is important for mitochondrial quality control, and is regulated by multiple proteins and pathways. ${ }^{34}$ It was found that depolarization of the mitochondrial membrane stabilizes PINK1 to the mitochondrial outer membrane, where it recruits and phosphorylates PARKIN/PARK2, an E3 ubiquitin protein ligase (Figure 3). ${ }^{35}$ The outer membrane GTPase Mitofusin 2 (MFN2) has been shown to have an important role in the recruitment of PARKIN to the mitochondria in a PINK1-dependent manner. PINK1 then phosphorylates MFN2 to induce its ubiquitination by PARKIN. ${ }^{36}$ Once recruited to the mitochondria, PARKIN ubiquitinates several mitochondrial substrates including voltage-dependent anion channel 1 (VDAC1) and MFN1/2.37,38 These modified proteins are then recognized by the adaptor protein SQSTM1/p62, which targets the damaged mitochondria to the autophagosomes based on its interaction with the ATG8 protein.

In addition to the involvement of PINK1 and PARKIN, depolarized mitochondria also recruit ATG9A and ULK1 independently to initiate the formation of the phagophore, a step which is followed by the recruitment of WIPI-1, ATG14, DFCP1, and ATG16L1 to induce the elongation of the membrane, before LC3 recruitment. Nonetheless, the substrate for ULK1 kinase has not been identified in this process

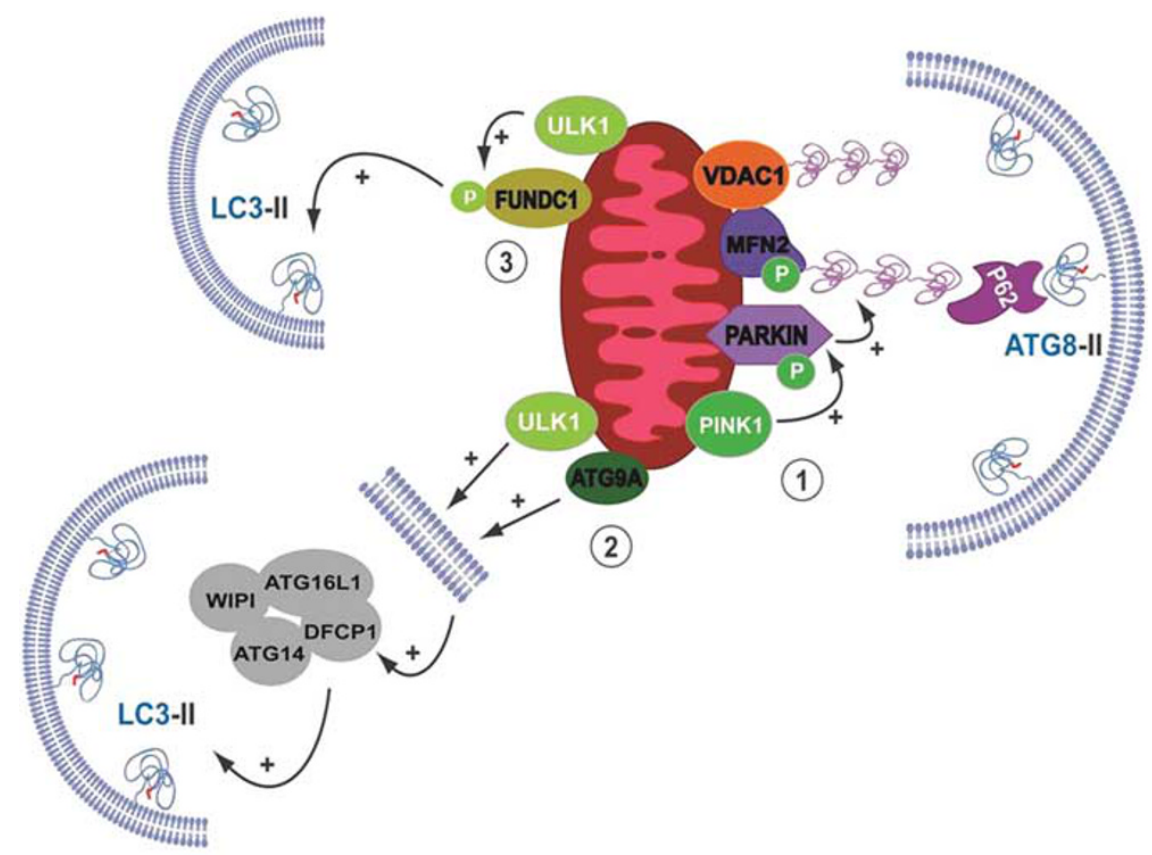

Figure 3 Phosphorylation of proteins involved in mitophagy. (1) In response to mitochondrial depolarization, PINK1 recruits PARKIN to the mitochondria, phosphorylates PARKIN, which then ubiquitinates several mitochondrial proteins, such as VDAC1 or MFN1/2. These modified proteins are recognized by the autophagy acceptor protein SQSTM1/P62, which targets the damaged mitochondria to the autophagosomes through its association with the ATG8 proteins. (2) In addition, ULK1 and ATG9A are recruited to depolarized mitochondria and induce the de novo formation of autophagosomes. This process also requires the involvement of the WIPI, ATG16L1, ATG14, and DFCP1 proteins and the LC3 protein to efficiently target the mitochondria into the autophagosomes. (3) Moreover, ULK1 is recruited to the damaged mitochondria, where it phosphorylates FUNDC1, inducing its association with LC3 and the efficient recruitment of mitochondria into the autophagosomes. 
(Figure 3). ${ }^{39}$ Hypoxia or mitochondrial uncoupling also induces upregulation and translocation of the kinase ULK1 to fragmented mitochondria where it binds and phosphorylates FUN14 Domain Containing 1 (FUNDC1) at Ser17. Interaction between ULK1 and FUNDC1 is required for ULK1 translocation to the mitochondria, and phosphorylation of FUNDC1 enhances FUNDC1 binding to LC3 to induce the targeting of mitochondria into autophagosomes for their degradation (Figure 3). ${ }^{40}$

\section{Phosphorylation of TFEB on the Lysosomal Membrane}

In addition to initiation of autophagy, MTORC1 also interacts with transcription factor EB (TFEB), and is thereby involved in lysosome recycling. Under normal conditions, MTORC1 colocalizes with and phosphorylates TFEB on the lysosomal membrane preventing its transport to the nucleus. Under starvation conditions, MTORC1 is inhibited, thereby releasing TFEB that can translocate to the nucleus to activate genes involved in lysosome biogenesis and autophagy, including LC3B, WIPI, ATG9B, and SQSTM1. ${ }^{41-43}$

\section{REGULATION OF AUTOPHAGY BY O-GLCNACYLATION}

In addition to being modified by phosphorylation, serine and threonine residues can also be modified by the O-linked attachment of $\beta$-N-acetyl-glucosamine (O-GlcNAc) (Figure 4a). Unlike classical O- and N-linked glycosylation, which is restricted to the endoplasmic reticulum and
Golgi apparatus, O-GlcNAcylation occurs on nuclear, cytoplasmic, and mitochondrial proteins. To date well over 1000 proteins have been characterized as O-GlcNAc targets, and it has also been shown to have an important role in modulating proteasome activity, ${ }^{44,45}$ signal transduction, ${ }^{46-49}$ nuclear transport, ${ }^{50}$ translation and transcription, ${ }^{51}$ and apoptosis. ${ }^{52,53}$ Uridine-diphosphate-N-acetylglucosamine (UDP-GlcNAc) is the essential sugar donor for the formation of O-GlcNAcylated proteins, which is catalyzed by O-GlcNAc transferase (OGT). Overall levels of protein O-GlcNAcylation are tightly regulated, with generation catalyzed by OGT, and removal catalyzed by $\beta-\mathrm{N}$-acetylglucosaminidase (OGA). Since serine and threonine residues are targets for both O-GlcNAcylation and phosphorylation, it is possible for them to compete with one another; however, there is growing evidence that there is a complex interaction between the two modifications. For example, increasing cellular O-GlcNAc levels through inhibition of OGA decreased phosphorylation at 280 sites, as well as increasing phosphorylation at 148 sites. ${ }^{54}$ The extensive interplay between O-GlcNAcylation and phosphorylation is also supported by the growing number of kinases that have been identified as O-GlcNAc targets, as well as the fact that OGT and OGA are known to form transient complexes with both kinases and phosphatases.

While alterations in O-GlcNAc levels have most commonly been associated with chronic disease models, such as diabetes

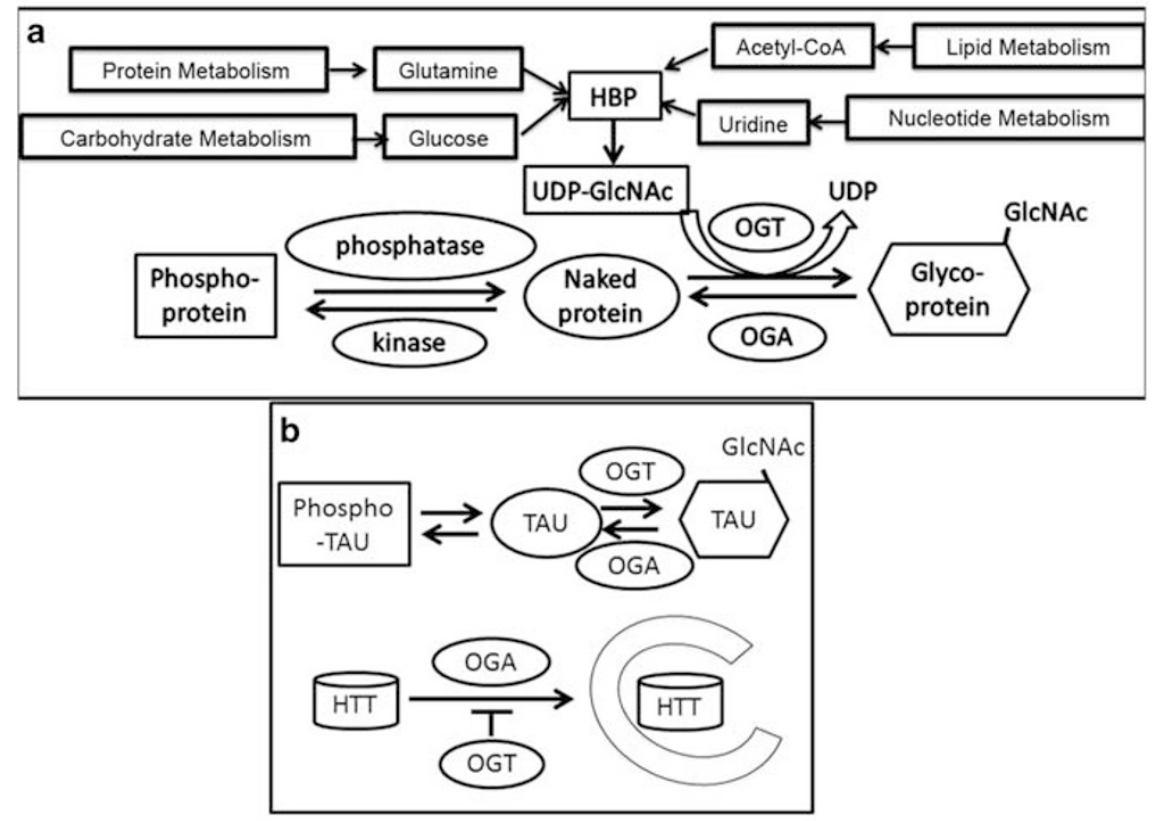

Figure 4 Regulation of autophagy by O-GIcNAcylation. (a) Uridine diphospho- $\beta$-N-acetylglucosamine (UDP-GlcNAc) is synthesized from glucose by hexose biosynthetic pathway (HBP), and acts as a source of monosaccharide $\mathrm{N}$-acetylglucosamine (GlcNAc). The enzymes and the biosynthetic pathways involved in the process are depicted. Covalent attachment of GlcNAc to nuclear and cytosolic proteins is referred to as O-GlcNAcylation, which is catalyzed by a single enzyme, O-GlcNAc transferase (OGT), and the removal catalyzed by O-GlcNAcase (OGA). (b) In Alzheimer's disease brain, TAU phosphorylation has been found to be inversely related to TAU O-GlcNAcylation. However, in Neuro2A cells, overexpression of OGA enhanced autophagy, decreased mutant HUNTINGTIN, and attenuated toxicity. Overexpression of OGT attenuated autophagy, and exacerbated mutant HUNTINGTIN accumulation and toxicity. 
and cancer, it is now clear that O-GlcNAc is essential for mammalian cell viability as evidenced by the embryonic lethality phenotypes of knockout mice deficient in either OGT or OGA. ${ }^{55,56}$ Moreover, acute activation of pathways resulting in increased protein $\mathrm{O}-\mathrm{GlcNAc}$ levels have been shown to improve the tolerance of cells to a wide range of stress stimuli. ${ }^{29}$ On the other hand, in C. elegans, OGA loss of function, which increased overall O-GlcNAcylation, enhanced the toxicity induced by TAU, $\beta$-amyloid, and polyglutamine; ${ }^{57}$ in contrast, OGT loss of function, which decreased O-GlcNAcylation, attenuated the same aggregate induced toxicity ${ }^{57}$ Interestingly, loss of either OGT or OGA led to the accumulation of autophagosomes; surprisingly, however, a role for O-GlcNAc cycling in regulating autophagy flux has not been examined. ${ }^{57}$ On the other hand, it has been shown that both BCL2 and BECN1, key regulators of autophagy, undergo O-GlcNAcylation, in response to nutrient deprivation. ${ }^{58}$ In addition, both AKT and AMPK, key regulators of the MTORC1 complex, are also targets for O-GlcNAcylation. ${ }^{59,60}$ Taken together, these observations provide strong support for a role of O-GlcNAcylation in regulating the autophagic response to stress.

In the context of neurodegenerative diseases in mammalian cells and rodent models, OGA is highly expressed at both the mRNA and protein levels in neurons and in human brains. ${ }^{61,62}$ Moreover, dysregulation in O-GlcNAc cycling increases with age, a known risk factor for development of neurodegenerative diseases. ${ }^{63,64}$ Among others, TAU is the most extensively studied O-GlcNAcylated protein in the brain because of the strong connection between its phosphorylation and Alzheimer's disease. In Alzheimer's disease brains, O-GlcNAcylation of TAU is decreased, and cells overexpressing OGT cDNA or shRNA, decreases and increases phosphorylation of TAU, respectively. ${ }^{65,66}$ Treatment of Alzheimer's disease mouse models with OGA inhibitors has been shown to increase O-GlcNAcylation and decrease phosphorylation of TAU both in vitro and in vivo (Figure 4b). ${ }^{67-69}$ To date, the role of O-GlcNAcylation in mediating the pathophysiology of Parkinson's disease has not been investigated; however, $\alpha$-synuclein is modified by $\mathrm{O}-$ GlcNAc and this attenuates its aggregation in vitro. ${ }^{70} \mathrm{In}$ contrast, it has recently been shown that suppression of O-GlcNAcylation by overexpression of OGA led to decreased SQSTM1/p62 and increased LC3-II, suggesting an enhanced autophagic flux. Azaserine inhibits glutamine fructose-6phosphate amidotransferase, one of the key enzymes of hexosamine biosynthesis pathway, and thereby leads to decreased O-GlcNAcylation and a decrease of LC3-II and SQSTM1/p62 in Neuro2A cells, attenuating toxicity by mutant HUNTINGIN exon 1 fragment in these cells. ${ }^{71}$

\section{REGULATION OF AUTOPHAGY BY UBIQUITIN-LIKE CONJUGATION AND UBIQUITINATION}

The expansion of the autophagosomal membrane involves the ubiquitination-like reactions of autophagy proteins
ATG8 and ATG12. E1-like protein ATG7 activates ATG8 and ATG12, which are then conjugated to phosphatidylethanolamine (PE) and ATG5, by E2-like proteins ATG3 and ATG10, respectively. Association of ATG16 with the ATG12ATG5 conjugates stimulates the ATG8-PE conjugation in a process that resembles E3-like activity (Figure 5a). ${ }^{72}$ Ubiquitination, by conjugating an $8-\mathrm{kDa}$ ubiquitin to proteins on lysine residues, can regulate autophagy activities. It has been shown that MTORC1 regulator DEPTOR, ULK1, BCL-2, BECN1, and BNIP1 can all be ubiquitinated. Ubiquitination of DEPTOR by Skp-cullin-F-box protein (SCF) in growth factor-rich conditions releases MTORC1 from DEPTOR inhibition and attenuates autophagy. ${ }^{73,74}$ ATG4B ubiquitination by RING finger protein 5 (RNF5) led to ATG4B degradation, which in turn affects LC3 turnover and limits autophagy. ${ }^{75}$ ULK1 ubiquitination by TRAF6 stabilizes the active ULK1 complex, therefore promoting autophagy. ${ }^{76}$ BECN1 ubiquitination by TRAF6 promotes TLR4-dependent autophagy, ${ }^{77}$ whereas BCL2 ubiquitination by PARKIN increases its interaction with BECN1 resulting in inhibition of autophagy. ${ }^{78}$ Also, BECN1 ubiquitination by neural precursor cell-expressed developmentally downregulated 4 (NEDD4) promotes BECN1 degradation and inhibits autophagy. ${ }^{79}$ BCL2/adenovirus E1B 19-kDa interacting protein 1 (BNIP1) ubiquitination by mitochondrial located E3 ligase RNF185 facilitates recruitment of p62 and LC3 (Figures 5b-e). ${ }^{80}$

The ubiquitin protein ligase E3 component N-recognin 4 (UBR4) has been shown to be highly expressed in endodermderived, autophagy-active cells in the yolk sac and has an essential role in embryonic development. Evidence suggests that UBR4 facilitates the delivery of intracellular organelles and macromolecules to the autophagosomes, although what specific proteins are UBR4 substrates in these autophagyactive cells and whether its ubiquitin ligase activities are required for these processes are unclear. ${ }^{81}$

Autophagy of the ribosomes, also known as ribophagy, depends on a rapid decrease of a $60 \mathrm{~S}$ ribosome-associated E3 ligase LTN1 in response to starvation, and a consequent decrease of ribosomal protein RPL25 ubiquitination. ${ }^{82}$ Mitophagy is also regulated by PARKIN-mediated ubiquitination. ${ }^{83}$ PARKIN is an E3 ubiquitin ligase that has been shown to ubiquitinate several mitochondrial proteins. Recruitment of PARKIN to the depolarized mitochondria has been shown to ubiquitinate MFN1 and MFN2, which are then degraded, facilitating mitophagy. ${ }^{84}$ PARKIN has also been shown to ubiquitinate VDAC1 in both HeLa and SHSY5Y cells, and the presence of VDAC1 is required for PARKIN/PINK1-mediated mitophagy in HeLa cells. ${ }^{37}$ VDAC1 ubiquitination alone is not responsible for mitochondrial depolarization-induced mitophagy in mouse embryonic fibroblasts (MEFs); ${ }^{85}$ however, in the absence of all three VDACs 1, 2, and 3, mitophagy is impaired in MEFs ${ }^{86}$ (Figure 5f).

MIRO1 and MIRO2 are outer mitochondrial membrane (OMM) proteins associated with coupling of mitochondria 
to the kinesin- and dynein-dependent microtubule transport pathway, thereby regulating mitochondrial trafficking and distribution. ${ }^{87,88}$ MIRO1 and MIRO proteins were found to be downregulated by PARKIN overexpression and CCCP treatment along with other PARKIN targets in HeLa, SH-SY5Y, and MEFs. ${ }^{89}$ Previously, it was reported that phosphorylation of MIRO at Serine 156 is important for the subsequent action of PARKIN, ${ }^{90}$ although a recent study has shown that MIRO ubiquitination in dopaminergic neuroblastoma cells is independent of phosphorylation at Serine 156, but is dependent on PINK1-dependent phosphorylation of PARKIN at Serine $65 .{ }^{91}$ In addition to MIROs, mitochon- drial hexokinase is also ubiquitinated by PARKIN. ${ }^{92,93}$ There is also evidence that in response to membrane depolarization, a pool of mitochondrial proteins, including TOM20 and FIS1, are degraded by PARKIN and ubiquitin-proteasomemediated mechanisms, both of which have essential roles in mitophagy. ${ }^{89}$ The landscape of the PARKIN-dependent ubiquitylome has recently been further expanded to include proteins involved in proteasome assembly, metabolism, and apoptosis. ${ }^{94}$ Despite these observations, whether ubiquitination of this broad spectrum of mitochondrial proteins is synergistic or antagonistic to one another in modulating mitophagy remains unclear (Figure 5f). Ubiquitination by
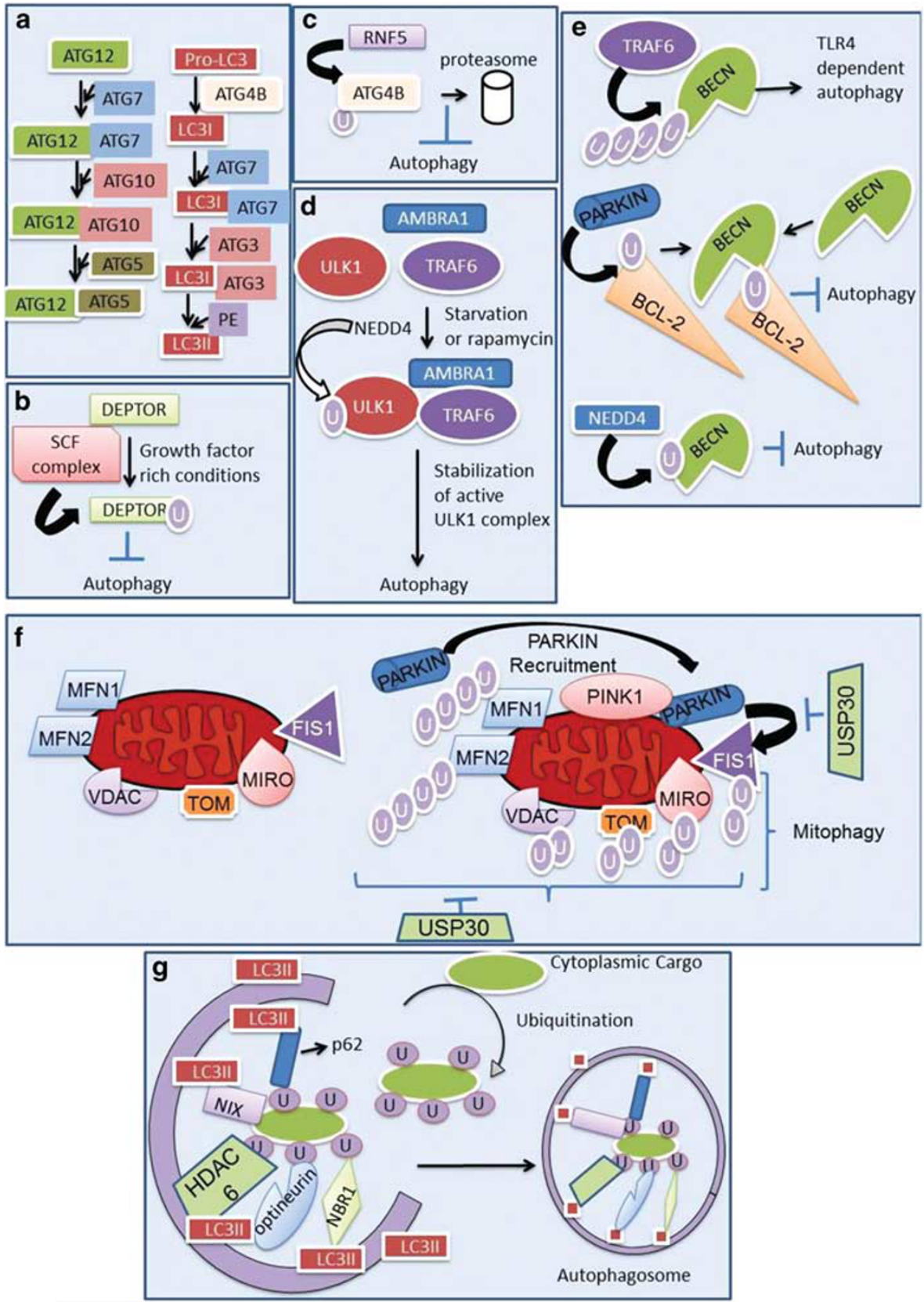
PARKIN has recently been shown to be modulated by an opposing activity from a mitochondrial deubiquitinase USP30. ${ }^{95}$

Besides PARKIN, other ubiquitin ligases may also have an important role in modifying proteins involved in mitophagy. Independent of PARKIN, MFN1 and MFN2 can also be ubiquitinated by glycoprotein 78 and glycoprotein 78-induced mitophagy is dependent on MFN1. ${ }^{96}$ Membraneassociated RING-CH-V (MARCH-V) was identified as a mitochondrial ubiquitin ligase that interacts with MFN2 and ubiquitinates DRP1 in COS7 cells, and MARCH-V overexpression was shown to promote long tubular mitochondria. $^{97}$ Interestingly, loss of MARCH-V also promotes mitochondrial elongation in an MFN1-dependent manner. In response to stress, MFN1, but not MFN2, induces mitochondrial elongation in HeLa cells. ${ }^{98}$ How MARCH-V is involved in regulating mitophagy is currently unclear (Figure 5).

Ubiquitin binding proteins have been shown to have an important role in bringing ubiquitinated proteins to the autophagosomes via their LIR. These ubiquitin binding proteins include SQSTM1/p62, neighbor of BRCA1 gene 1 (NBR1), and histone deacetylase 6 (HDAC6), the nuclear dot protein $52 \mathrm{kDa}$ (NDP52), and optineurin (OPTN) which is involved in aggrephagy. ${ }^{99-101}$ SQSTM1/p62 also interacts with TRIM50, TRAF6, MURF2, and KEAP1, which facilitates ubiquitination of target proteins and their subsequent degradation. ${ }^{102-106}$ OPTN and NDP52 have been shown to have important roles in xenophagy. ${ }^{107} \mathrm{BH} 3$-only family protein NIX, ${ }^{108,109}$ BCL2/adenovirus E1B 19-kDa interacting protein 3 (BNIP3), ${ }^{110}$ and FUN14 domain containing 1 (FUNDC1) ${ }^{111}$ have also been shown to be important for mitophagy by recognizing mitochondria that contain extensively ubiquitinated outer membrane proteins (Figure $5 \mathrm{~g}$ ).

\section{REGULATION OF AUTOPHAGY BY ACETYLATION}

Regulatory components of the autophagy pathway are also controlled by lysine acetylation, which involves the attach- ment of at least one acetyl group; generally at the $\mathrm{N}$-terminus of the protein. Nutrient starvation rapidly depletes acetylcoenzyme A that consequently results in lower overall acetylation of cytoplasmic proteins, which is associated with an increase in autophagy. ${ }^{112,113}$ Knockdown of acetyl-coenzyme A synthetase in Drosophila brains has also been shown to enhance autophagy and lifespan. ${ }^{112}$

Acetylation and de-acetylation of histones and nonhistone proteins have been reported to have an important role in autophagy regulation. Cell protective induction of autophagy by starvation or inhibition of MTORC1 led to downregulation of the histone acetyltransferase hMOF/ KAT8/MYST1 and consequent de-acetylation of histone H4 lysine 16 (H4K16ac). Fifty-five autophagy-related genes, including those that encode LC3 and ULK1, exhibited altered expression levels as assessed by a global run-on-sequencing assay, as well as decreased acetylated $\mathrm{H} 4 \mathrm{~K} 16$ (H4K16ac) as quantified by chromatin immunoprecipitation-sequencing (ChIP-seq) assay (Figure 6a). ${ }^{114}$

Starvation-induced microtubule hyperacetylation also promotes autophagy and cell survival. In this case, microtubule hyperacetylation is mediated by $\alpha$-tubulin acetyltransferase-1 ( $\alpha$ TAT-1/MEC-17), whose activity is inhibited by p300 and activated by AMPK. ${ }^{115}$ How microtubule hyperacetylation promotes autophagy is unclear, but it was proposed that microtubule acetylation might impact its interaction with microtubule-associated proteins, including the LC3 family of proteins (Figure 6b).

Acetylation of autophagy proteins also has an important role in autophagic flux. Acetyltransferase p300 has been shown to acetylate ATG5, ATG7, LC3, and ATG12 proteins, thereby inhibiting autophagy. ${ }^{116}$ In cells deprived of growth factors, glycogen synthase (GSK-3) phosphorylates acetyltransferase TIP60 on Ser86 leading to its activation. TIP60 in turn activates ULK1 by acetylation, which has an important role in autophagy induction. ${ }^{117}$ In addition, LC3B-II has been shown to be markedly deacetylated during serum

Figure 5 Ubiquitination by E3 ligases of autophagy proteins has an important role in regulating autophagy. (a) Ubiquitin-like conjugation system in autophagy. Expansion of the phagophore depends on two ubiquitin-like conjugation systems. Conjugation of ATG5 to ATG12 requires the E1 enzyme ATG7 and the E2 enzyme ATG10, generates an oligomeric complex between the ATG12-ATG5 conjugate and ATG16L1. ATG8/LC3 proteins are subsequently conjugated to phosphatidylethanolamine (PE) following cleavage by the cysteine protease ATG4 acting on nascent ATG8s (proLC3) to expose a C-terminal glycine residue required for covalent attachment to PE. The exposed glycine of ATG8 (LC3-I) is activated by ATG7 (E1), then transferred to ATG3 (E2-like enzyme) forming an ATG8 ATG3 thioester intermediate, before ATG8 is conjugated to PE by the E3-like ATG12-ATG5ATG16 complex. (b) Under conditions of high nutrient and growth factor availability, SCF (Skp1-Cullin1-F-box protein) E3 ligase complex triggers ubiquitination of DEPTOR, which in turn leads to its degradation and inhibits autophagy. (c) Basal autophagy is regulated by membrane-associated E3 ligase RNF5 by controlling cysteine protease ATG4B levels. ATG4B is ubiquitinated under basal conditions by RNF5, the degradation of ATG4B in turn leads to insufficient LC3 processing, thereby inhibiting autophagy. (d) In response to autophagic stimulus, TRAF6 ubiquitinates ULK1 thereby increasing ULK1 kinase activity resulting in autophagy induction. (e) BECN and BCL-2 ubiquitination status differentially regulate autophagy. While TRAF6mediated polyubiquitination of BECN leads to autophagic induction, PARKIN mediated monoubiquitination of BCL-2 results in stabilization of BECNBCL2 complex leading to inhibition of autophagy, and BECN ubiquitination by NEDD4 also suppresses autophagy. (f) Ubiquitination of substrates by PARKIN mediates mitophagy. In response to stress induction, PINK1 is stabilized at the mitochondrial outer membrane, and recruits PARKIN, which then ubiquitinates MFN1, MFN2, VDAC, TOM, FIS1, and MIRO, resulting in induction of mitophagy. Mitochondrial deubiquitinase USP30 removes ubiquitin from proteins that are ubiquitinated by PARKIN, thus attenuating PARKIN-mediated mitophagy. (g) The cargo for selective autophagy is recruited to the autophagosomes by autophagy adaptors, such as SQSTM1/P62, neighbor of BRCA1 gene 1 (NBR1), histone deacetylase 6 (HDAC6), autophagy receptor BH3-only family protein NIX, the ubiquitin receptor nuclear dot protein 52kd (NDP52), and optineurin, that are associated both with the cargo and with lipidated ATG8/LC3 (LC3II), linking ubiquitin-tagged cargo with the autophagic machinery. 


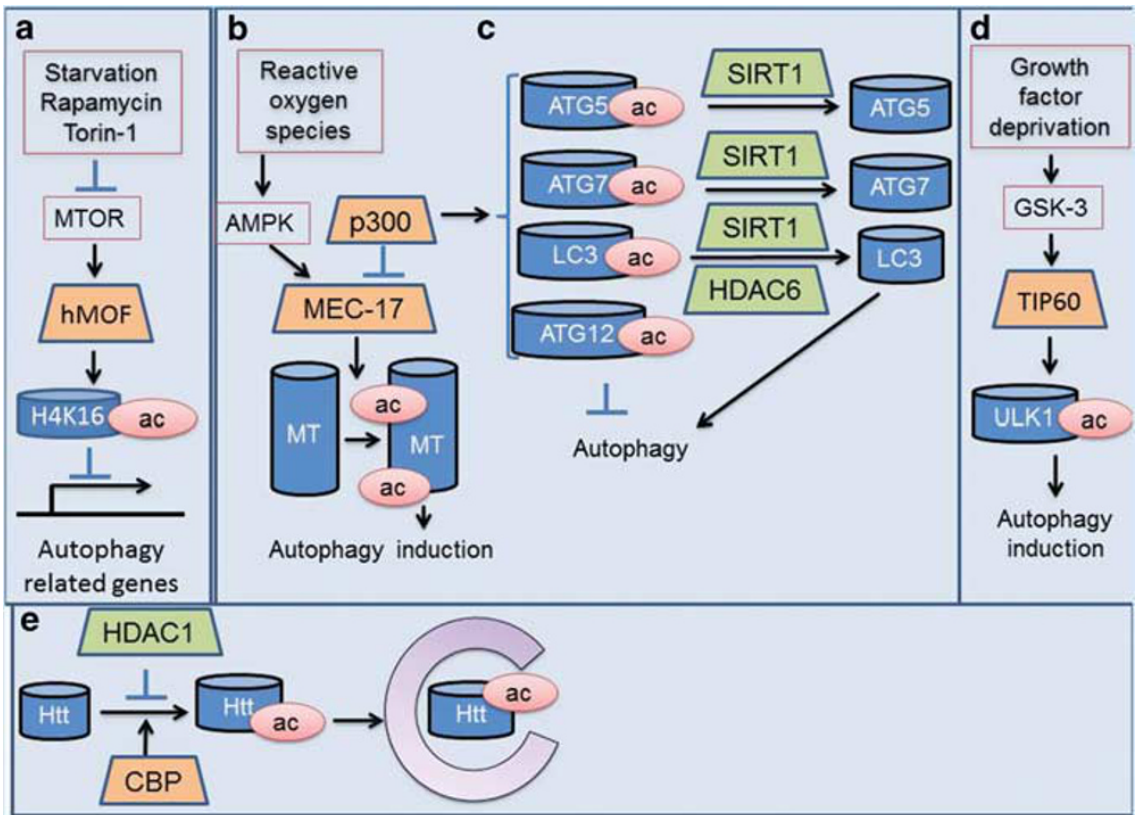

Figure 6 Autophagy regulation by acetylation. (a) Transcription regulation of autophagy genes can be regulated by H4K16 deacetylation via inhibition of MTOR and consequent inhibition of hMOF acetyltransferase. (b) Autophagy induction can also be promoted by microtubule acetylation, which is mediated by $\alpha$-tubulin acetyltransferase- 1 (alphaTAT-1/MEC-17) which senses reactive oxygen species and starvation by AMPK and p300 mediated mechanisms. (c) It was reported that deacetylation of autophagy proteins by SIRT1 or HDAC6 promotes, and acetylation of autophagy proteins by p300 inhibits autophagy. (d) ULK1 acetylation mediated by acetyltransferase TIP60 which senses growth factor deprivation also promotes autophagy. (e) CBP-mediated mutant huntingtin acetylation promotes its degradation by autophagic activities and HDAC1 mediated mutant huntingtin deacetylation inhibits its degradation.

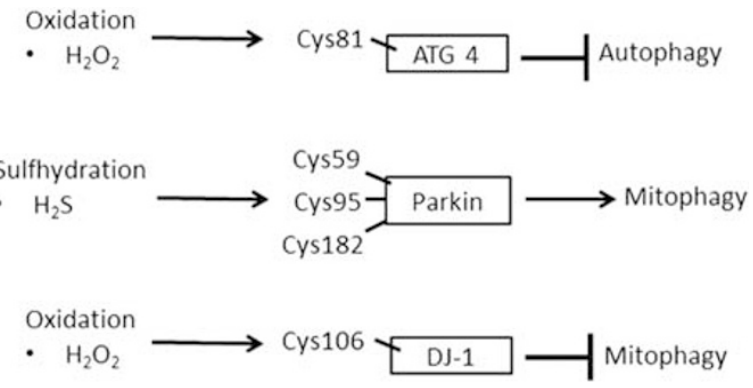

Figure 7 Regulation of autophagy by thiol modification. (1) ATG4 activity has been shown to be regulated by hydrogen peroxide modification of Cys81; 125 (2) Mitophagy-related protein PARKIN can be modified by sulfhydration at Cys59, 95, and 182, and thus decrease its inactivating nitrosation and enhance its function in neuroprotection. (3) Oxidation of Cys106 on DJ-1 decreases its activity and mitochondrial localization.

deprivation-induced autophagy in HeLa cells. Inhibition of HDAC6 by an HDAC6 inhibitor tubacin, or HDAC6, by siRNA knockdown, resulted in increased acetylation of LC3B-II, along with impaired degradation of SQSTM1/p62, suggesting a link between acetylation status of LC3B-II and autophagic clearance. ${ }^{118}$ SIRT1, a mammalian deacetylase has been shown to deacetylate several ATG proteins, such as
ATG5, ATG7, and LC3, in response to starvation. The absence of SIRT1 resulted in impaired autophagy leading to increased levels of SQSTM1/p62 and inhibition of autophagosome formation (Figures $6 \mathrm{c}$ and d). ${ }^{119}$

Post-translational modifications of target proteins can also modulate their autophagic clearance. Inhibition of HDAC1 or activation of CBP-mediated acetylation have been shown to increase the acetylation of mutant HUNTINTIN (HTT) resulting in its increased clearance, and decreased neurotoxicity. ${ }^{120}$ These studies demonstrate that acetylation influences autophagy at multiple levels (Figure 6e).

\section{ROLE OF THIOL MODIFICATIONS IN AUTOPHAGY REGULATION}

Redox-sensitive proteins have highly reactive thiol groups on cysteine residues (R-SH) that allows for modification by a variety of nucleophilic reactive species, and particularly susceptible to increased modification during increased oxidative or nitrosative stress. ${ }^{121,122}$ Thiol modifications on key cysteine residues have been proposed to serve as a regulatory mechanism that controls the autophagy-lysosomal pathway. ${ }^{6,123,124}$ Recombinant ATG4 activity has been shown to be regulated by hydrogen peroxide $\left(\mathrm{H}_{2} \mathrm{O}_{2}\right)$ modification of a cysteine residue near the active site (Cys81). ${ }^{125}$ Mutations to either of two highly conserved cysteines in PARKIN, whose loss of function has been linked to Parkinson's disease, 
result in loss of PARKIN folding and activity, and dysfunctional mitophagy. ${ }^{37,126}$ PARKIN cysteines (Cys 59, Cys 95, and Cys182) can also be modified by hydrogen sulfide $\left(\mathrm{H}_{2} \mathrm{~S}\right)$, resulting in sulfhydration, which can compete with pathogenic nitrosation of the same residues to enhance PARKIN activity and neuroprotective effects. ${ }^{127}$ Similarly, DJ-1, another Parkinson's disease-related protein linked to mitophagy, also has a key cysteine residue (Cys106) required for both its antioxidant properties and translocation to the mitochondria. ${ }^{128}$ These studies demonstrate the importance of cysteines in proper autophagic function, and that increased production of reactive species could modify key autophagy proteins altering their function (Figure 7).

\section{Conclusions and Future Directions}

Post-translational modification of autophagy proteins has emerged as an essential regulatory mechanism. As most proteins contain modifiable serine, threonine, lysine, or cysteine residues, they are subjected to conditions that favor modifications on these residues in response to nutrient availability, growth factor deprivation, hyperoxia, and hypoxia, as well as generation and propagation of reactive species. One key remaining question is how the proteome of diverse post-translational modifications on multiple autophagy proteins impacts general and selective autophagic flux. Furthermore, it is important to recognize cell type and tissuespecific regulation of these post-translational modifications, and how the specificities are achieved at the level of enzymes that add or delete these modifications. Understanding which amino-acid residues in autophagy proteins are modified, and identifying the situations that lead to their modifications, could provide key therapeutic targets in mitigating harmful pathologies associated with diseases. ${ }^{129}$

\section{ACKNOWLEDGMENTS}

This work was supported by HL101192, HL-110366 (JC), and NIHR01-NS064090 (JZ)

\section{DISCLOSURE/CONFLICT OF INTEREST}

The authors declare no conflict of interest.

1. Finley D, Ciechanover A, Varshavsky A. Ubiquitin as a central cellular regulator. Cell 2004;116:2.

2. Moremen KW, Tiemeyer M, Nairn AV. Vertebrate protein glycosylation: diversity, synthesis and function. Nat Rev Mol Cell Biol 2012:13:448-462.

3. Resh MD. Targeting protein lipidation in disease. Trends Mol Med 2012;18:206-214.

4. Bononi A, Agnoletto C, De ME, et al. Protein kinases and phosphatases in the control of cell fate. Enzyme Res 2011;2011:329098.

5. Yorimitsu T, Klionsky DJ. Autophagy: molecular machinery for selfeating. Cell Death Differ 2005;12(Suppl 2):1542-1552.

6. Dodson M, Darley-Usmar V, Zhang J. Cellular metabolic and autophagic pathways: traffic control by redox signaling. Free Radic Biol Med 2013;63:207-221.

7. Efeyan A, Zoncu R, Sabatini DM. Amino acids and mTORC1: from lysosomes to disease. Trends Mol Med 2012;18:524-533.

8. Wirth M, Joachim J, Tooze SA. Autophagosome formation-the role of ULK1 and Beclin1-PI3KC3 complexes in setting the stage. Semin Cancer Biol 2013;23:301-309.
9. Gwinn DM, Shackelford DB, Egan DF, et al. AMPK phosphorylation of raptor mediates a metabolic checkpoint. Mol Cell 2008;30: 214-226.

10. Egan DF, Shackelford DB, Mihaylova MM, et al. Phosphorylation of ULK1 (hATG1) by AMP-activated protein kinase connects energy sensing to mitophagy. Science 2011;331:456-461.

11. Kundu M. ULK1, mammalian target of rapamycin, and mitochondria: linking nutrient availability and autophagy. Antioxid Redox Signal 2011;14:1953-1958.

12. Cantley LC. The phosphoinositide 3-kinase pathway. Science 2002 296:1655-1657.

13. Vander $\mathrm{HE}$, Lee $\mathrm{SI}$, Bandhakavi $\mathrm{S}$, et al. Insulin signalling to mTOR mediated by the Akt/PKB substrate PRAS40. Nat Cell Biol 2007;9: 316-323.

14. Sancak $Y$, Thoreen CC, Peterson TR, et al. PRAS40 is an insulin-regulated inhibitor of the mTORC1 protein kinase. Mol Cell 2007:25:903-915.

15. Panaretou C, Domin J, Cockcroft $S$, et al. Characterization of p150, an adaptor protein for the human phosphatidylinositol (Ptdlns) 3-kinase. Substrate presentation by phosphatidylinositol transfer protein to the p150.Ptdins 3-kinase complex. J Biol Chem 1997;272: 2477-2485.

16. Itakura E, Mizushima N. Characterization of autophagosome formation site by a hierarchical analysis of mammalian Atg proteins. Autophagy 2010;6:764-776.

17. Simonsen A, Birkeland HC, Gillooly DJ, et al. Alfy, a novel FYVEdomain-containing protein associated with protein granules and autophagic membranes. J Cell Sci 2004;117:4239-4251.

18. Kang R, Zeh HJ, Lotze MT, et al. The Beclin 1 network regulates autophagy and apoptosis. Cell Death Differ 2011;18:571-580.

19. Wei $Y$, Pattingre S, Sinha S, et al. JNK1-mediated phosphorylation of Bcl-2 regulates starvation-induced autophagy. Mol Cell 2008;30: 678-688.

20. Zalckvar E, Berissi $H$, Eisenstein $M$, et al. Phosphorylation of Beclin 1 by DAP-kinase promotes autophagy by weakening its interactions with $\mathrm{BCl}-2$ and $\mathrm{BCl}-\mathrm{XL}$. Autophagy 2009;5:720-722.

21. Wang RC, Wei $Y, A n Z$, et al. Akt-mediated regulation of autophagy and tumorigenesis through Beclin 1 phosphorylation. Science 2012;338:956-959.

22. Shirahama-Noda K, Kira S, Yoshimori T, et al. TRAPPIII is responsible for vesicular transport from early endosomes to Golgi, facilitating Atg9 cycling in autophagy. J Cell Sci 2013;126:4963-4973.

23. He C, Baba M, Cao $Y$, et al. Self-interaction is critical for Atg9 transport and function at the phagophore assembly site during autophagy. Mol Biol Cell 2008;19:5506-5516.

24. Young AR, Chan EY, Hu XW, et al. Starvation and ULK1-dependent cycling of mammalian Atg9 between the TGN and endosomes. J Cell Sci 2006;119:3888-3900.

25. Papinski $D$, Schuschnig $M$, Reiter $W$, et al. Early steps in autophagy depend on direct phosphorylation of Atg9 by the Atg1 kinase. Mol Cell 2014;53:471-483.

26. Okazaki N, Yan J, Yuasa S, et al. Interaction of the Unc-51-like kinase and microtubule-associated protein light chain 3 related proteins in the brain: possible role of vesicular transport in axonal elongation. Brain Res Mol Brain Res 2000;85:1-12.

27. Cherra III SJ, Kulich SM, Uechi G, et al. Regulation of the autophagy protein LC3 by phosphorylation. J Cell Biol 2010;190:533-539.

28. Jiang $\mathrm{H}$, Cheng $\mathrm{D}$, Liu $\mathrm{W}$, et al. Protein kinase $\mathrm{C}$ inhibits autophagy and phosphorylates LC3. Biochem Biophys Res Commun 2010;395: 471-476.

29. Colecchia D, Strambi A, Sanzone S, et al. MAPK15/ERK8 stimulates autophagy by interacting with LC3 and GABARAP proteins 1 . Autophagy 2012;8:1724-1740.

30. Alemu EA, Lamark T, Torgersen KM, et al. ATG8 family proteins act as scaffolds for assembly of the ULK complex: sequence requirements for LC3-interacting region (LIR) motifs. J Biol Chem 2012;287: 39275-39290.

31. Matsumoto G, Wada K, Okuno M, et al. Serine 403 phosphorylation of p62/SQSTM1 regulates selective autophagic clearance of ubiquitinated proteins. Mol Cell 2011;44:279-289.

32. Mao K, Chew LH, Inoue-Aono Y, et al. Atg29 phosphorylation regulates coordination of the Atg17-Atg31-Atg29 complex with the Atg11 scaffold during autophagy initiation 2. Proc Natl Acad Sci USA 2013;110:E2875-E2884. 
33. Kabeya Y, Noda NN, Fujioka Y, et al. Characterization of the Atg17Atg29-Atg31 complex specifically required for starvation-induced autophagy in Saccharomyces cerevisiae. Biochem Biophys Res Commun 2009;389:612-615.

34. Hill BG, Benavides GA, Lancaster Jr. JR, et al. Integration of cellular bioenergetics with mitochondrial quality control and autophagy. Biol Chem 2012;393:1485-1512.

35. Matsuda N, Sato S, Shiba K, et al. PINK1 stabilized by mitochondrial depolarization recruits Parkin to damaged mitochondria and activates latent Parkin for mitophagy. J Cell Biol 2010;189:211-221.

36. Chen Y, Dorn GW. PINK1-phosphorylated mitofusin 2 is a Parkin receptor for culling damaged mitochondria. Science 2013;340:471-475.

37. Geisler S, Holmstrom KM, Skujat D, et al. PINK1/Parkin-mediated mitophagy is dependent on VDAC1 and p62/SQSTM1. Nat Cell Biol 2010;12:119-131.

38. Gegg ME, Cooper JM, Chau KY, et al. Mitofusin 1 and mitofusin 2 are ubiquitinated in a PINK1/parkin-dependent manner upon induction of mitophagy. Hum Mol Genet 2010;19:4861-4870.

39. Itakura E, Kishi-Itakura C, Koyama-Honda I, et al. Structures containing Atg9A and the ULK1 complex independently target depolarized mitochondria at initial stages of Parkin-mediated mitophagy. J Cell Sci 2012;125:1488-1499.

40. Wu W, Tian W, Hu Z, et al. ULK1 translocates to mitochondria and phosphorylates FUNDC1 to regulate mitophagy. EMBO Rep 2014;15: 566-575.

41. Settembre C, Di MC, Polito VA, et al. TFEB links autophagy to lysosomal biogenesis. Science 2011;332:1429-1433.

42. Settembre C, Zoncu R, Medina DL, et al. A lysosome-to-nucleus signalling mechanism senses and regulates the lysosome via mTOR and TFEB. EMBO J 2012;31:1095-1108.

43. Martina JA, Chen $Y$, Gucek $M$, et al. MTORC1 functions as a transcriptional regulator of autophagy by preventing nuclear transport of TFEB. Autophagy 2012;8:903-914.

44. Han I, Kudlow JE. Reduced O glycosylation of $\mathrm{Sp} 1$ is associated with increased proteasome susceptibility. Mol Cell Biol 1997;17: 2550-2558.

45. Zachara NE, Hart GW. O-GlcNAc modification: a nutritional sensor that modulates proteasome function. Trends Cell Biol 2004;14: 218-221.

46. Zachara NE, Hart GW. O-GlcNAc a sensor of cellular state: the role of nucleocytoplasmic glycosylation in modulating cellular function in response to nutrition and stress. Biochim Biophys Acta 2004;1673: 13-28.

47. Slawson C, Housley MP, Hart GW. O-GlcNAc cycling: how a single sugar post-translational modification is changing the way we think about signaling networks. J Cell Biochem 2006;97:71-83.

48. Darley-Usmar VM, Ball LE, Chatham JC. Protein O-linked beta-Nacetylglucosamine: a novel effector of cardiomyocyte metabolism and function. J Mol Cell Cardiol 2012;52:538-549.

49. Love DC, Hanover JA. The hexosamine signaling pathway: deciphering the "O-GlcNAc code". Sci STKE 2005;2005:re13.

50. Guinez C, Lemoine J, Michalski JC, et al. 70-kDa-heat shock protein presents an adjustable lectinic activity towards O-linked $\mathrm{N}$-acetylglucosamine. Biochem Biophys Res Commun 2004;319:21-26.

51. Comer Fl, Hart GW. O-GlcNAc and the control of gene expression. Biochim Biophys Acta 1999;1473:161-171.

52. Liu K, Paterson AJ, Zhang F, et al. Accumulation of protein O-GlcNAc modification inhibits proteasomes in the brain and coincides with neuronal apoptosis in brain areas with high O-GlcNAc metabolism. J Neurochem 2004;89:1044-1055.

53. Wells L, Whelan SA, Hart GW. O-GICNAc: a regulatory post-translational modification. Biochem Biophys Res Commun 2003;302:435-441.

54. Wang Z, Gucek M, Hart GW. Cross-talk between GlcNAcylation and phosphorylation: site-specific phosphorylation dynamics in response to globally elevated O-GlcNAc. Proc Natl Acad Sci USA 2008;105:13793-13798.

55. Shafi R, lyer SP, Ellies LG, et al. The O-GlcNAc transferase gene resides on the $X$ chromosome and is essential for embryonic stem cell viability and mouse ontogeny. Proc Natl Acad Sci USA 2000;97:5735-5739.

56. Yang $Y R$, Song $M$, Lee $H$, et al. O-GlcNAcase is essential for embryonic development and maintenance of genomic stability. Aging Cell 2012;11:439-448.
57. Wang $\mathrm{P}$, Lazarus $\mathrm{BD}$, Forsythe $\mathrm{ME}$, et al. O-GlcNAc cycling mutants modulate proteotoxicity in Caenorhabditis elegans models of human neurodegenerative diseases. Proc Natl Acad Sci USA 2012;109:17669-17674.

58. Marsh SA, Powell PC, Dell'italia LJ, et al. Cardiac O-GlcNAcylation blunts autophagic signaling in the diabetic heart. Life Sci 2013;92: 648-656.

59. Bullen JW, Balsbaugh JL, Chanda D, et al. Cross-talk between two essential nutrient-sensitive enzymes: O-GlcNAc transferase (OGT) and AMP-activated protein kinase (AMPK). J Biol Chem 2014;289: 10592-10606.

60. Wang $S$, Huang $X$, Sun $D$, et al. Extensive crosstalk between O-GlcNAcylation and phosphorylation regulates Akt signaling. PLoS ONE 2012;7:e37427.

61. Akimoto $\mathrm{Y}, \mathrm{Comer} \mathrm{Fl}$, Cole RN, et al. Localization of the O-GlcNAc transferase and O-GlcNAc-modified proteins in rat cerebellar cortex. Brain Res 2003;966:194-205.

62. Gao Y, Wells L, Comer Fl, et al. Dynamic O-glycosylation of nuclear and cytosolic proteins: cloning and characterization of a neutral, cytosolic beta-N-acetylglucosaminidase from human brain. J Biol Chem 2001;276:9838-9845.

63. Fulop $N$, Feng $W$, Xing $D$, et al. Aging leads to increased levels of protein O-linked $\mathrm{N}$-acetylglucosamine in heart, aorta, brain and skeletal muscle in Brown-Norway rats. Biogerontology 2008;9:139-151.

64. Liu $\mathrm{Y}, \mathrm{Li} \mathrm{X}, \mathrm{Yu} \mathrm{Y}$, et al. Developmental regulation of protein O-GlcNAcylation, O-GIcNAc transferase, and O-GlcNAcase in mammalian brain. PLoS ONE 2012;7:e43724.

65. Robertson LA, Moya KL, Breen KC. The potential role of tau protein O-glycosylation in Alzheimer's disease. J Alzheimers. Dis 2004;6: 489-495.

66. Liu F, Shi J, Tanimukai H, et al. Reduced O-GlcNAcylation links lower brain glucose metabolism and tau pathology in Alzheimer's disease. Brain 2009;132:1820-1832.

67. Yuzwa SA, Shan X, Macauley MS, et al. Increasing O-GlcNAc slows neurodegeneration and stabilizes tau against aggregation. Nat Chem Biol 2012;8:393-399.

68. Yuzwa SA, Macauley MS, Heinonen JE, et al. A potent mechanisminspired O-GlcNAcase inhibitor that blocks phosphorylation of tau in vivo. Nat Chem Biol 2008;4:483-490.

69. Yu Y, Zhang L, Li X, et al. Differential effects of an O-GlcNAcase inhibitor on tau phosphorylation. PLoS ONE 2012;7:e35277.

70. Marotta NP, Cherwien CA, Abeywardana T, et al. O-GlcNAc modification prevents peptide-dependent acceleration of alpha-synuclein aggregation. Chembiochem 2012;13:2665-2670.

71. Kumar A, Singh PK, Parihar R, et al. Decreased O-linked GlcNAcylation protects from cytotoxicity mediated by huntingtin exon1 protein fragment. J Biol Chem 2014;289:13543-13553.

72. Hanada T, Noda NN, Satomi Y, et al. The Atg12-Atg5 conjugate has a novel E3-like activity for protein lipidation in autophagy. J Biol Chem 2007;282:37298-37302.

73. Zhao $Y$, Xiong $X$, Sun $Y$. DEPTOR, an mTOR inhibitor, is a physiological substrate of SCF(betaTrCP) E3 ubiquitin ligase and regulates survival and autophagy. Mol Cell 2011;44:304-316.

74. Gao D, Inuzuka $H$, Tan MK, et al. mTOR drives its own activation via SCF(betaTrCP)-dependent degradation of the mTOR inhibitor DEPTOR. Mol Cell 2011;44:290-303.

75. Kuang $E$, Okumura CY, Sheffy-Levin $S$, et al. Regulation of ATG4B stability by RNF5 limits basal levels of autophagy and influences susceptibility to bacterial infection. PLoS Genet 2012;8:e1003007.

76. Nazio F, Strappazzon F, Antonioli M, et al. mTOR inhibits autophagy by controlling ULK1 ubiquitylation, self-association and function through AMBRA1 and TRAF6. Nat Cell Biol 2013;15:406-416.

77. Shi CS, Kehrl JH. TRAF6 and A20 regulate lysine 63-linked ubiquitination of Beclin-1 to control TLR4-induced autophagy. Sci Signal 2010;3:ra42.

78. Chen D, Gao F, Li B, et al. Parkin mono-ubiquitinates BCl-2 and regulates autophagy. J Biol Chem 2010;285:38214-38223.

79. Platta HW, Abrahamsen H, Thoresen SB, et al. Nedd4-dependent lysine-11-linked polyubiquitination of the tumour suppressor Beclin 1. Biochem J 2012;441:399-406.

80. Tang F, Wang B, Li N, et al. RNF185, a novel mitochondrial ubiquitin E3 ligase, regulates autophagy through interaction with BNIP1. PLoS ONE 2011;6:e24367. 
81. Tasaki T, Kim ST, Zakrzewska A, et al. UBR box N-recognin-4 (UBR4), an $\mathrm{N}$-recognin of the $\mathrm{N}$-end rule pathway, and its role in yolk sac vascular development and autophagy. Proc Natl Acad Sci USA 2013;110:3800-3805.

82. Ossareh-Nazari B, Nino CA, Bengtson $\mathrm{MH}$, et al. Ubiquitylation by the Ltn1 E3 ligase protects 60S ribosomes from starvation-induced selective autophagy. J Cell Biol 2014;204:909-917.

83. Jin SM, Youle RJ. PINK1- and Parkin-mediated mitophagy at a glance. J Cell Sci 2012;125:795-799.

84. Tanaka A, Cleland MM, Xu S, et al. Proteasome and p97 mediate mitophagy and degradation of mitofusins induced by Parkin. J Cell Biol 2010;191:1367-1380.

85. Narendra D, Kane LA, Hauser DN, et al. p62/SQSTM1 is required for Parkin-induced mitochondrial clustering but not mitophagy; VDAC1 is dispensable for both. Autophagy 2010;6:1090-1106.

86. Sun $\mathrm{Y}$, Vashisht AA, Tchieu J, et al. Voltage-dependent anion channels (VDACs) recruit Parkin to defective mitochondria to promote mitochondrial autophagy. J Biol Chem 2012;287:40652-40660.

87. Macaskill AF, Rinholm JE, Twelvetrees AE, et al. Miro1 is a calcium sensor for glutamate receptor-dependent localization of mitochondria at synapses. Neuron 2009;61:541-555.

88. Russo GJ, Louie K, Wellington A, et al. Drosophila Miro is required for both anterograde and retrograde axonal mitochondrial transport. J Neurosci 2009;29:5443-5455.

89. Chan NC, Salazar AM, Pham AH, et al. Broad activation of the ubiquitin-proteasome system by Parkin is critical for mitophagy. Hum Mol Genet 2011;20:1726-1737.

90. Wang X, Winter D, Ashrafi G, et al. PINK1 and Parkin target Miro for phosphorylation and degradation to arrest mitochondrial motility. Cell 2011;147:893-906.

91. Birsa N, Norkett R, Wauer T, et al. K27 ubiquitination of the mitochondrial transport protein Miro is dependent on serine 65 of the Parkin ubiquitin ligase. J Biol Chem 2014:289:14569-14582.

92. McCoy MK, Kaganovich A, Rudenko IN, et al. Hexokinase activity is required for recruitment of parkin to depolarized mitochondria. Hum Mol Genet 2014;23:145-156.

93. Okatsu K, lemura S, Koyano F, et al. Mitochondrial hexokinase HKI is a novel substrate of the Parkin ubiquitin ligase. Biochem Biophys Res Commun 2012;428:197-202.

94. Sarraf SA, Raman M, Guarani-Pereira V, et al. Landscape of the PARKIN-dependent ubiquitylome in response to mitochondrial depolarization. Nature 2013;496:372-376.

95. Bingol B, Tea JS, Phu L, et al. The mitochondrial deubiquitinase USP30 opposes parkin-mediated mitophagy. Nature 2014;510: 370-375.

96. Fu M, St-Pierre $\mathrm{P}$, Shankar J, et al. Regulation of mitophagy by the Gp78 E3 ubiquitin ligase. Mol Biol Cell 2013;24:1153-1162.

97. Nakamura $N$, Kimura $Y$, Tokuda $M$, et al. MARCH-V is a novel mitofusin 2- and Drp1-binding protein able to change mitochondrial morphology. EMBO Rep 2006;7:1019-1022.

98. Park YY, Lee S, Karbowski M, et al. Loss of MARCH5 mitochondrial E3 ubiquitin ligase induces cellular senescence through dynaminrelated protein 1 and mitofusin 1. J Cell Sci 2010;123:619-626.

99. Kuang E, Qi J, Ronai Z. Emerging roles of E3 ubiquitin ligases in autophagy. Trends Biochem Sci 2013:38:453-460.

100. McEwan DG, Dikic I. The Three Musketeers of Autophagy: phosphorylation, ubiquitylation and acetylation. Trends Cell Biol 2011; 21:195-201.

101. Rogov V, Dotsch V, Johansen T, et al. Interactions between autophagy receptors and ubiquitin-like proteins form the molecular basis for selective autophagy. Mol Cell 2014;53:167-178.

102. Fusco $C$, Micale L, Egorov M, et al. The E3-ubiquitin ligase TRIM50 interacts with HDAC6 and p62, and promotes the sequestration and clearance of ubiquitinated proteins into the aggresome. PLoS ONE 2012;7:e40440.

103. Jain A, Lamark T, Sjottem E, et al. p62/SQSTM1 is a target gene for transcription factor NRF2 and creates a positive feedback loop by inducing antioxidant response element-driven gene transcription. J Biol Chem 2010;285:22576-22591

104. Komatsu M, Kurokawa $\mathrm{H}$, Waguri $\mathrm{S}$, et al. The selective autophagy substrate p62 activates the stress responsive transcription factor Nrf2 through inactivation of Keap1. Nat Cell Biol 2010;12:213-223.
105. Lange S, Xiang F, Yakovenko A, et al. The kinase domain of titin controls muscle gene expression and protein turnover. Science 2005:308:1599-1603.

106. Lau A, Wang XJ, Zhao F, et al. A noncanonical mechanism of Nrf2 activation by autophagy deficiency: direct interaction between Keap1 and p62. Mol Cell Biol 2010;30:3275-3285.

107. Wild P, Farhan H, McEwan DG, et al. Phosphorylation of the autophagy receptor optineurin restricts Salmonella growth. Science 2011;333:228-233.

108. Schweers RL, Zhang J, Randall MS, et al. NIX is required for programmed mitochondrial clearance during reticulocyte maturation. Proc Natl Acad Sci USA 2007:104:19500-19505.

109. Sandoval H, Thiagarajan P, Dasgupta SK, et al. Essential role for Nix in autophagic maturation of erythroid cells. Nature 2008;454: 232-235

110. Zhang J, Ney PA. Role of BNIP3 and NIX in cell death, autophagy, and mitophagy. Cell Death Differ 2009;16:939-946.

111. Liu L, Feng D, Chen G, et al. Mitochondrial outer-membrane protein FUNDC1 mediates hypoxia-induced mitophagy in mammalian cells. Nat Cell Biol 2012;14:177-185.

112. Eisenberg T, Schroeder S, Andryushkova A, et al. Nucleocytosolic depletion of the energy metabolite acetyl-coenzyme a stimulates autophagy and prolongs lifespan. Cell Metab 2014;19:431-444.

113. Marino G, Pietrocola F, Eisenberg T, et al. Regulation of autophagy by cytosolic acetyl-coenzyme A. Mol Cell 2014;53:710-725.

114. Fullgrabe J, Lynch-Day MA, Heldring $\mathrm{N}$, et al. The histone $\mathrm{H} 4$ lysine 16 acetyltransferase hMOF regulates the outcome of autophagy. Nature 2013;500:468-471.

115. Mackeh $\mathrm{R}$, Lorin $\mathrm{S}$, Ratier $\mathrm{A}$, et al. Reactive oxygen species, AMP-activated protein kinase, and the transcription cofactor p300 regulate alpha-Tubulin Acetyltransferase-1 (alphaTAT-1/MEC-17)dependent microtubule hyperacetylation during cell stress. J Biol Chem 2014:289:11816-11828.

116. Lee IH, Finkel T. Regulation of autophagy by the p300 acetyltransferase. J Biol Chem 2009:284:6322-6328.

117. Lin SY, Li TY, Liu Q, et al. GSK3-TIP60-ULK1 signaling pathway links growth factor deprivation to autophagy. Science 2012;336:477-481.

118. Liu KP, Zhou D, Ouyang DY, et al. LC3B-II deacetylation by histone deacetylase 6 is involved in serum-starvation-induced autophagic degradation. Biochem Biophys Res Commun 2013;441:970-975.

119. Lee IH, Cao L, Mostoslavsky R, et al. A role for the NAD-dependent deacetylase Sirt1 in the regulation of autophagy. Proc Natl Acad Sci USA 2008;105:3374-3379.

120. Jeong $\mathrm{H}$, Then $\mathrm{F}$, Melia Jr. TJ, et al. Acetylation targets mutant huntingtin to autophagosomes for degradation. Cell 2009;137: 60-72.

121. Murphy MP. Mitochondrial thiols in antioxidant protection and redox signaling: distinct roles for glutathionylation and other thiol modifications. Antioxid Redox Signal 2012;16:476-495.

122. Anathy V, Roberson EC, Guala AS, et al. Redox-based regulation of apoptosis: S-glutathionylation as a regulatory mechanism to control cell death. Antioxid Redox Signal 2012;16:496-505.

123. Lee J, Giordano S, Zhang J. Autophagy, mitochondria and oxidative stress: cross-talk and redox signalling. Biochem J 2012;441:523-540.

124. Levonen $A L$, Hill BG, Kansanen $E$, et al. Redox regulation of antioxidants, autophagy, and the response to stress: Implications for electrophile therapeutics. Free Radic Biol Med 2014;71C:196-207.

125. Scherz-Shouval R, Shvets E, Fass E, et al. Reactive oxygen species are essential for autophagy and specifically regulate the activity of Atg 4 . EMBO J 2007;26:1749-1760.

126. Hampe $\mathrm{C}$, Ardila-Osorio $\mathrm{H}$, Fournier $\mathrm{M}$, et al. Biochemical analysis of Parkinson's disease-causing variants of Parkin, an E3 ubiquitinprotein ligase with monoubiquitylation capacity. Hum Mol Genet 2006;15:2059-2075.

127. Vandiver MS, Paul BD, $\mathrm{Xu} R$, et al. Sulfhydration mediates neuroprotective actions of parkin. Nat Commun 2013;4:1626.

128. Krebiehl G, Ruckerbauer S, Burbulla LF, et al. Reduced basal autophagy and impaired mitochondrial dynamics due to loss of Parkinson's disease-associated protein DJ-1. PLoS ONE 2010;5:e9367.

129. Giordano S, Darley-Usmar V, Zhang J. Autophagy as an essential cellular antioxidant pathway in neurodegenerative disease. Redox Biol 2014;2:82-90. 